\title{
Issues in contemporary fluid management
}

Jean-Louis Vincent

\author{
Université Libre de Bruxelles, Brussels, Belgium
}

\begin{abstract}
Fluid management strategies need to be guided by an understanding of the pathophysiologic mechanisms underlying fluid imbalance. In the hypovolaemic patient, reduced circulating blood volume and venous return and, in severe cases, altered tissue perfusion may initiate a cascade of pathophysiologic processes culminating in multiple organ failure. The objectives of fluid management are to maintain adequate blood pressure, tissue oxygenation and intravascular fluid volume. Both crystalloids and colloids can be useful for these purposes. In the hypovolaemic patient with normal pulmonary function, the use of colloids to maintain colloid osmotic pressure can limit the development of peripheral as well as pulmonary oedema. However, choice of fluid is less important in states of increased lung capillary permeability. Further evidence is needed to broaden understanding of the optimal roles for particular fluid management strategies. Experimental models can make an important contribution in gathering such evidence. Rigorous pharmacoeconomic studies are also needed to define the benefits and costs of differing fluid regimens.
\end{abstract}

Keywords: colloids, hypotension, sepsis, serum albumin, shock

Most critically ill patients will require volume expansion at some juncture during their stay in the intensive care unit (ICU). Common indications for fluid administration in the ICU include trauma, haemorrhage, dehydration and sepsis. Surgical patients also typically require fluids both perioperatively and postoperatively. An array of additional clinical situations may also prompt fluid administration; for instance, in burn victims, hypoproteinaemic patients, cirrhotic patients with ascites undergoing therapeutic paracentesis, and so on.

The pathophysiologic mechanisms of fluid imbalance and the optimal approaches to fluid management continue to be actively investigated in both clinical and preclinical studies.
Further light is also being shed on fluid management issues by recent meta-analyses. The meta-analytic findings are provocative; however, many questions have been raised regarding their implications for clinical practice. One such question is the appropriateness of relying on mortality as the primary outcome measure, since choice of fluid may exert a relatively minor impact on this particular endpoint. Also, the manner in which the skilled clinician implements a fluid management strategy may have a greater bearing on outcome than the type of fluid administered.

The appropriate basis for adopting a particular fluid management strategy is an understanding of the underlying pathophysiology of fluid imbalance. Hypovolaemia can

$\mathrm{ALI}=$ acute lung injury; $\mathrm{ARDS}=$ acute respiratory distress syndrome; ICU = intensive care unit. 
lead to reduced circulating blood volume, diminished venous return and, in severe cases, arterial hypotension. The aims of fluid management are to preserve intravascular fluid volume, adequate blood pressure and tissue oxygenation. In the hypovolaemic patient with normal pulmonary function, the use of colloids to maintain colloid osmotic pressure can limit the development of pulmonary oedema under conditions of elevated hydrostatic pressure. This approach may be of more limited value during states of increased lung capillary permeability; however, since the permeability barrier generally remains at least partially intact, colloid administration may still be useful. There is growing appreciation that hypovolaemia may also precipitate localized microcirculatory disorders, in which tissue perfusion is locally compromised despite apparent systemic normovolaemia. Severe hypovolaemia may ultimately lead to multiple organ failure.

The appropriate roles for colloids and crystalloids continue to be a major focus of clinical investigation. These two fluid categories appear to be largely indistinguishable in terms of their effects on preload recruitable stroke volume or oxygen delivery. However, because of their propensity for leakage into the extravascular space, crystalloids need to be administered in volumes approximately three-fold greater than those of colloids in order to achieve comparable resuscitation endpoints. Colloids, in contrast, are retained to a greater degree in the intravascular space, and hence smaller infused volumes are needed to attain haemodynamic endpoints. Based on limited data, colloids may offer the advantage of enhancing oxygen consumption to a greater extent than crystalloids. Crystalloids are generally regarded as first-line fluids for the haemodynamically stable patient and, in the typical scenario, colloids are administered in addition to rather than in lieu of crystalloids. However, when the patient is haemodynamically compromised, colloids are preferred by many clinicians including authorities in the field of critical care medicine.

The comparative advantages and limitations of natural and artificial colloids are also a topic of contention. Advantages of the natural colloid albumin vis-à-vis artificial colloids include a less limited recommended dose range, reduced risk of haemostatic complications, clearance without tissue deposition, and lower incidence of anaphylactoid reactions. Albumin is also readily monitored on a routine basis, so that there is diminished potential for excessive administration of this colloid and consequent hyperalbuminaemia, although this risk is remote. Artificial colloids are less expensive per unit dose and have not been subject to the same periodic supply shortages as albumin.

Clinical investigations into the pathophysiology of fluid imbalance have become increasingly sophisticated with the availability of monitoring tools such as intrathoracic blood volume measurement, mercury plethysmography and labelled erythrocytes, plasma or albumin. Nevertheless, an important role remains to be played by experimental models. There are, of course, limitations in the use of animal models. Suitable models of multiple organ failure and of acute respiratory distress syndrome (ARDS), for example, have proven elusive. Nevertheless, many models are available that can potentially reproduce a particular state of fluid imbalance and allow the effects of therapeutic interventions to be meaningfully assessed. Animal models can aid in the justification and design of clinical trials and are also well suited for investigating pathophysiologic mechanisms.

Intriguing new clinical data are now emerging in the area of acute lung injury (ALI) and ARDS. It is well established that hydrostatic pressure can contribute to ALI and ARDS. Transit of large molecules into the interstitial space may be increased due to capillary leakage in these inflammatory conditions. Nonetheless, the permeability barrier to large molecules is usually not entirely abolished, and therefore osmotic pressure may also play a role. A preliminary report has appeared describing the results of a randomized controlled trial employing combination therapy with albumin and furosemide in ALI patients. This therapy increased serum albumin and total serum protein levels, promoted weight loss, increased acute oxygenation and improved long-term haemodynamic stability without clinically important adverse effects. If these observations are confirmed in larger-scale trials, this fluid management approach could potentially provide a welcome option for these high-risk patients.

Although cost has long been a major concern in fluid management, the available data on which to base clinical decisions are highly limited. Most studies reported to date have focused exclusively on cost differences between administered fluids. Rigorous pharmacoeconomic data are needed that address the comparative total costs of care between different fluid management regimens in particular clinical indications. Specifically, the costs incurred as a result of fluid-associated morbidity, mortality and differences in length of ICU or hospital stay also need to be taken into account.

The optimal types of fluids and administration regimens for particular indications remain far from resolved. Fluid management controversies are long standing, especially concerning the comparative merits of colloids and crystalloids. Nevertheless, numerous studies are continuing to elucidate further the effectiveness and safety of differing fluid management approaches. As a consequence, this area of investigation remains vibrant.

Author affiliation: Department of Intensive Care, Université Libre de Bruxelles, Brussels, Belgium

Correspondence: Jean-Louis Vincent, MD, PhD, FCCM, Head, Department of Intensive Care, Hôpital Erasme, Université Libre de Bruxelles, Route de Lennik 808, B-1070 Brussels, Belgium. Tel: +32 2555 3380; fax: +32 2555 4555;

e-mail: jlvincen@ulb.ac.be 